\title{
A Cross Ratio Invariability-based RANSAC Registration Method
}

\author{
Jian $\mathrm{Liu}^{\text {a) }}$, Wenjun $\mathrm{Wu}^{\text {b) }}$ \\ College of Information and Control Engineering, Shenyang Jianzhu University, Shenyang 110168, China. \\ a)644630900@qq.com, ${ }^{\text {b) } 827003429 @ q q . c o m ~}$
}

\begin{abstract}
To improve registration performance of machine vision under complex environment, an improved RANSAC rapid point cloud registration algorithm is presented upon the principle of cross ratio invariability for internal feature points of a registered target. By analyzing the model of a registered target, the method can be used to work out the affine matrix between reference point cloud and target point cloud; cross ratio invariability of internal point data is taken as the constraint condition for registration to correct and remove feature points with wrong registration; besides, the time and accuracy of correct registration between a target image and a reference image are taken as the standards for registration effect evaluation. Lastly, it is indicated by comparing the algorithm and a traditional registration algorithm SAC-IA+ICP that the algorithm presented in the paper is substantially superior to the traditional registration algorithm, since it shows good robustness in large-scale 3D point cloud registration and significantly improved registration efficiency upon guaranteed precision.
\end{abstract}

Key words: Cross Ratio Invariability, 3D Point Cloud, Consensus Algorithm, Affine Matrix.

\section{INTRODUCTION}

Machine vision technology has developed rapidly in recent years, and practical application of 3D point cloud data has increasingly become a hot issue in machine vision researches. Currently, 3D point cloud data processing technology has been extensively applied in reverse engineering, computer vision, digitization of cultural relic and other fields. Rough registration technology is used to register two-point clouds with relatively large position difference; for example, the RANSAC [1] algorithm presented by Fischer realizes registration without considering point cloud position difference but does not apply to large-scale point cloud registration due to high algorithm complexity, long operation time, poor registration accuracy and low registration efficiency. Precise registration technology is used for point clouds processed by rough registration; for instance, best et al. [2] presented the ICP [3-10] algorithm; Zhang et al. [2] improved the ICP algorithm by introducing the Kid-tree algorithm; Granger et al. [2] applied the EM (Expectation Maximization) algorithm in the ICP algorithm; Gold et al. [4] raised a Soft assign registration algorithm; and Greenspan et al [10] utilized approximate k-d tree to search for closest registration points. These algorithms have been improved in searching for feature points but fail to remove and correct unrelated point and wrongly matched point. In view of abovementioned problems, an improved RANSAC rapid point cloud registration algorithm is presented with improved registration speed, precision and time.

\section{CROSS RATIO INVARIABILITY-BASED IMPROVED RANSAC ALGORITHM}

Specific steps for registration of target point cloud with the cross-ratio invariability-based RANSAC improved algorithm are presented below.

(1) It is assumed to randomly select a set of $\mathrm{n}$ matching points as the reference samples; $\mathrm{N}$ is the total data point amount; and $\theta$ is the possibility that the $\mathrm{n}$ data points fit the model, namely 


$$
\theta=\frac{n}{N}
$$

(2) We can obtain the reference model $M$ via the above reference samples; use all data points to test the model parameter and about the inside point amount $n^{\prime}$.

(3) Preset an error threshold $\delta$; for a case below $\delta$, the model area is the feature point area of the target cloud; for a case above $\delta$, the feature point area of the target cloud shall be recomputed.

(4) According to the gradient value of two feature points in the selected target point cloud area, the coordinate of the number $i$ feature point in the source point cloud image is set as $\left(x_{i}, y_{i}\right), i=1,2, \ldots, n$; the coordinate of the central feature point is $\left(x_{c}, y_{c}\right)$; and the distance between the two feature points can be expressed as:

$$
d_{i j}=\sqrt{\left(x_{i}-x_{j}\right)^{2}+\left(y_{i}-y_{j}\right)^{2}} \mathrm{i}, \mathrm{j}=1,2, \ldots, \mathrm{n} .
$$

The coordinate distance matrix of $n$ feature points in the source point cloud image is:

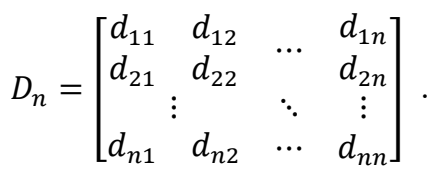

The threshold for coordinate distance between the central point of the target cloud and the central point of the source cloud is set to evaluate registration error; when the distance is larger than the set threshold, repeat abovementioned step (3) to step (4) until the condition of the set threshold is met.

(5) It is assumed that there are $m$ points with correct position and $n$ points with wrong position in the registered target image. $r, s, t$ Are 3 random, different and collinear points among the $m$ correctly positioned points, while $v$ is a point among the $n$ wrongly positioned points and collinear with $r, s, t . r, s, t, v$ Map to the 1st, 2nd, 3rd and 4 th points in the source point cloud respectively. According to Theorem 1, we can obtain

$$
\frac{d_{13}}{d_{23}}: \frac{d_{14}}{d_{24}}=\frac{d_{r t}}{d_{s t}}: \frac{d_{r v}}{d_{s v}}
$$

The coordinate point of wrongly registered target point cloud can be corrected according to the principle of cross ratio invariability, and the corrected point can be reevaluated. If the corrected point meets the requirement, move on to the next step, otherwise repeat step (5).

(6) After repeated operations for K times, select 6 pairs of corresponding points from the corrected matching points, and compute the initial value $\mathrm{H}$ of the rotation and translation matrix.

$$
\left[\begin{array}{cccc}
h_{1} & h_{2} & h_{3} & h_{4} \\
h_{5} & h_{6} & h_{7} & h_{8} \\
h_{9} & h_{10} & h_{11} & h_{12} \\
0 & 0 & 0 & 1
\end{array}\right]\left[\begin{array}{c}
x_{j} \\
y_{j} \\
z_{j} \\
1
\end{array}\right]=\left[\begin{array}{c}
x_{j}^{\prime} \\
y_{j}^{\prime} \\
z_{j}^{\prime} \\
1
\end{array}\right]
$$

In the formula, $\left(x_{j}^{\prime}, y_{j}^{\prime}, z_{j}^{\prime}\right)$ is a point in the target point cloud, while $\left(x_{j}, y_{j}, z_{j}\right)$ is corresponding to $\left(x_{j}^{\prime}, y_{j}^{\prime}, z_{j}^{\prime}\right)$ in the source point cloud.

\section{EXPERIMENTAL RESULT AND ANALYSIS}

To demonstrate the feasibility of the algorithm under complex scene, a registration experiment is conducted for in-garage vehicle models. Kinect was used for on-spot shooting in the experiment; initial vehicle point cloud images 
were obtained after de-nosing with Geomatics algorithm; and the registration experiment was conducted then. The collected vehicle model point cloud amounts and precision registration parameters are shown in Table 1.

TABLE 1. Experimental Parameters

\begin{tabular}{cccccc}
\hline \multicolumn{2}{l}{ Point Cloud Amount (Count) } & \multicolumn{4}{c}{ ICP Precise Registration Parameter } \\
\hline $\begin{array}{c}\text { Source Point } \\
\text { Cloud }\end{array}$ & $\begin{array}{c}\text { Target Point } \\
\text { Cloud }\end{array}$ & Threshold & $\begin{array}{c}\text { Maximum } \\
\text { Iteration }\end{array}$ & $\begin{array}{c}\text { Transformational Matrix } \\
\text { Difference }\end{array}$ & $\begin{array}{c}\text { Mean Square } \\
\text { Error }\end{array}$ \\
145994 & 162635 & 1.5 & Amount & De-10 & 0.2 \\
\hline
\end{tabular}

The data presented in Fig. 1 is the registration result based on the traditional algorithm (SAC-IA+ICP); in the Figure, the source point cloud is the white point cloud, the target cloud is green, and the rotated and translated cloud is red. The Fig. 2 presents the point cloud registration result based on the improved RANSAC algorithm, including the source point cloud in red, the target cloud in green and the rotated and translated cloud in blue. For Fig. 1 and Fig. 2, the (a), (b) and (c) figures are registration images of vehicle failure, (a) for vehicle with lifted front wheels, (b) for vehicle rollover and (c) for inversed vehicle, while the (d) figures are registration images of normal vehicle condition.

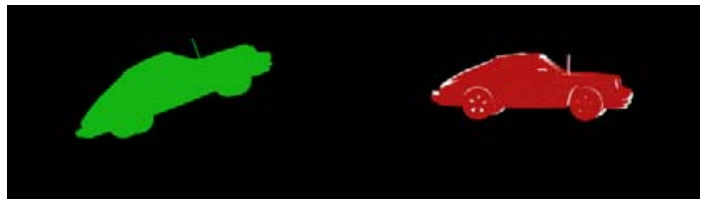

(a) Failure Image 1

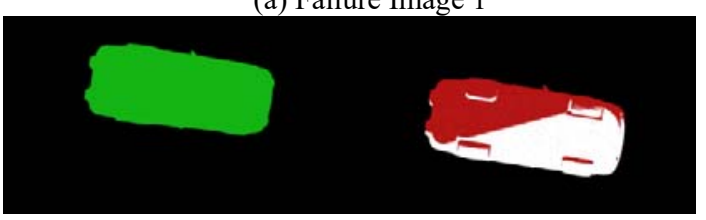

(c) Failure Image 3

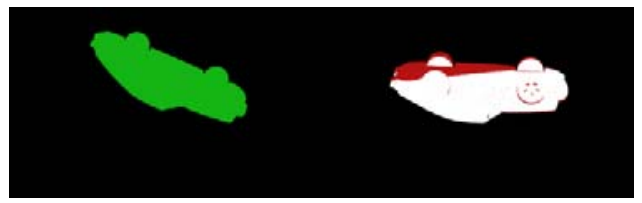

(b) Failure Image 2

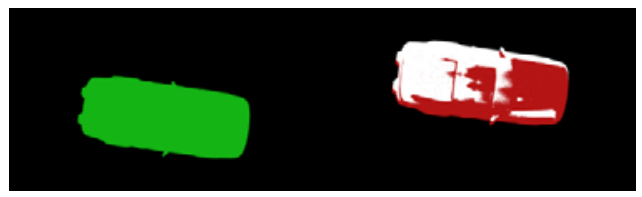

(d) Normal Image 4

FIGURE 1. Registration result based on SAC-IA+ICP.

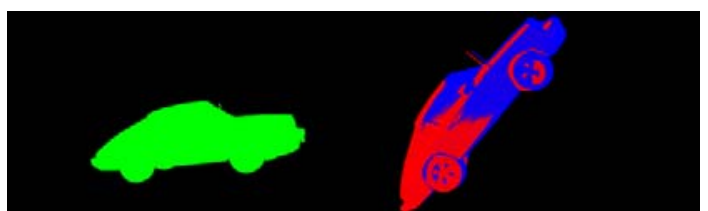

(a) Failure Image 1

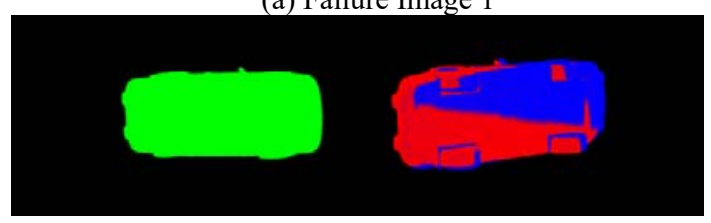

(c) Failure Image 3

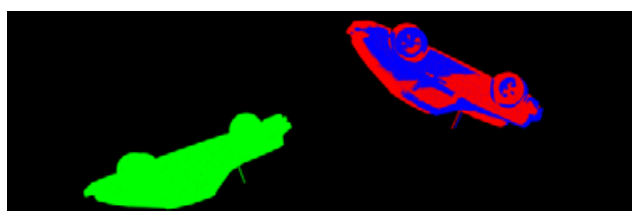

(b) Failure Image 2

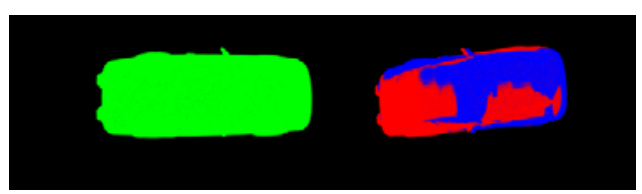

(d) Normal Image 4

FIGURE 2. Registration result based on the algorithm presented in the paper.

It can be viewed by comparing Fig. 1 and Fig. 2 that the traditional point cloud registration algorithm shows relatively good registration in the (a) figure, but the algorithm presented in the paper shows registration in a more global way in the (b), (c) and (d) figures. The algorithm presented in the paper completes matching by searching close point pairs, corrects wrongly matched points, significantly increases the amount of close point pair, frees the registration course from being limited to a certain area, expands the course to global form of a target point cloud, and makes registration results more comprehensive and intact. 
It can be viewed from Table 2 that the rough registration and the average times are far shorter in the improved RANSAC algorithm group than in the SAC-IA+ICP algorithm, and the rotation angles at all directions are all smaller in the former group. All these reflect the superior performance of the algorithm presented in the paper.

TABLE 2. Experimental Parameters.

\begin{tabular}{|c|c|c|c|c|}
\hline \multirow{2}{*}{} & \multicolumn{2}{|c|}{ SAC-IA+ICP } & \multicolumn{2}{c|}{ The algorithm presented in the paper } \\
\cline { 2 - 5 } & $\begin{array}{c}\text { Rough Registration } \\
\text { Time(s) }\end{array}$ & $\begin{array}{c}\text { Average Registration } \\
\text { Time (s) }\end{array}$ & $\begin{array}{c}\text { Rough Registration } \\
\text { Time(s) }\end{array}$ & $\begin{array}{c}\text { Average Registration } \\
\text { Time (s) }\end{array}$ \\
\hline car & 41.758 & 45.219 & 29.461 & 32.758 \\
\hline
\end{tabular}

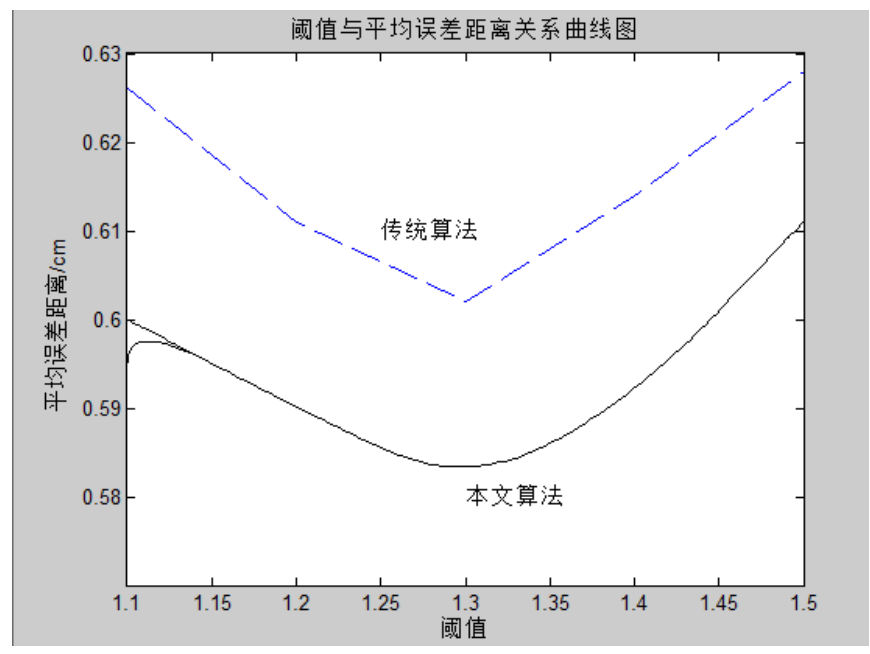

(a) Relations between threshold and average error distance

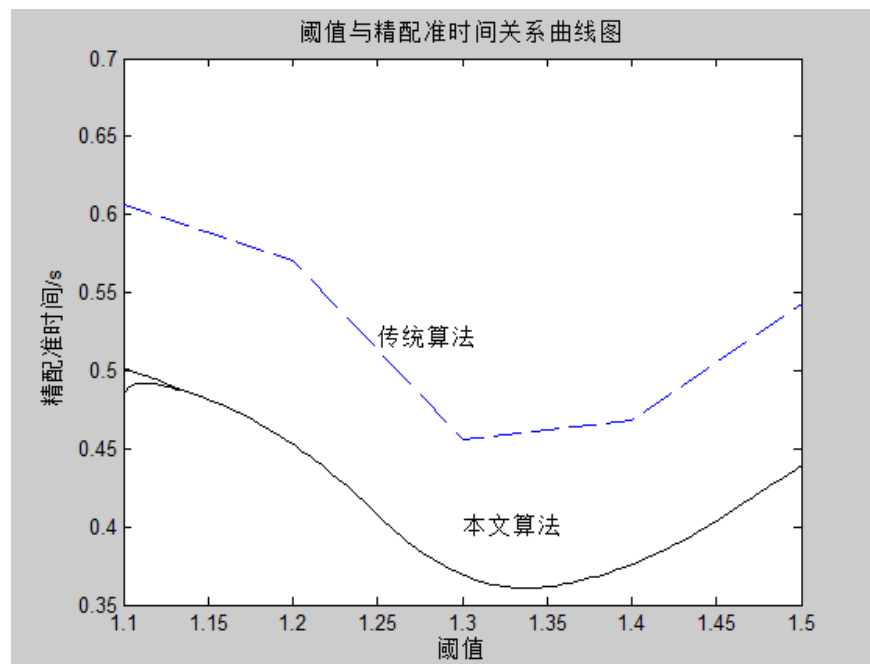

(b) Relations between threshold and precise registration time

FIGURE 3. Curves of relations between threshold and average error distance and between threshold and precise registration time.

In Fig. 3(a), the threshold is in direct proportion to the registration precision at first; with the reduction of threshold, the registration error declines, reaches a minimum level when the threshold is about 1.3, and then starts to increase. In Fig. 3(b), the threshold is directly proportional to the registration speed; the precise registration time is reduced with the threshold and reaches a minimal level at a threshold of about 1.3, and then increases. The precise registration times, rotation angles at $\mathrm{X} / \mathrm{Y} / \mathrm{Z}$ directions during registration courses and registration matching errors have been 
measured for 5 different threshold groups. It can be suggested from Fig. 3 that registration of vehicle model side views with the algorithm provided in the paper shows relatively smaller rotation angles at $\mathrm{X} / \mathrm{Y} / \mathrm{Z}$ direction during registration and an average registration error distance of below $0.611 \mathrm{~cm}$ and has a smallest average error distance and shortest precise registration time when the threshold is about 1.3.

\section{CONCLUSION}

To solve frequently occurred problems concerning error registration points and unrelated registration point during point cloud registration, a cross ratio invariability-based RANSAC rapid point cloud registration algorithm is presented in the paper. It is indicated by the experiments that the improved RANSAC rapid point cloud registration algorithm, by correcting wrongly matched point pairs and removing unrelated matching point pairs, reduces registration time in complex scene and registration error distance, being significant for high-efficient and precise realization of a point cloud registration course.

\section{REFERENCES}

1. Wang R Research on Camera Calibration and Point Cloud Registration Technology in Computer Vision[D]. Indian University,2015:9-14.

2. Best P J. A Method for Registration of 3-D Shapes [J]. IEEE Transactions on Pattern Analysis and Machine Intelligence, 1992, 14(3):239-256.

3. ZHANG Z Iterative Point Matching for Registration of Free-From Curves and Surfaces [J]. International Journal of Computer Vision, 1994, 13(2):119-152.

4. Granger Spanned X, Multi-scale EM-ICP: A Fast and Robust Approach for Surface Registration[C]. In Proctor 7th European Conference on Computer Vision(ECCV2002),2002:418-432.

5. Rusinkiewicz S, Levoy Efficient Variants of the ICP Algorithm[C].3DIM, IEEE Computer Society,2001:145152.

6. Men Research on Point Cloud Registration Method Based on Sampled Ball and ICP Algorithm [D]. Tsinghua University, 2012:7-8.

7. Zheng Y, Zhang M. Point cloud automatic registration technology based on improved ICP algorithm[J]. Control engineering, 2014,21(01):37-40.

8. Zeng F Xylem Lydia X P. Research on the Registration of Iterative Nearest Neighbor Algorithm Based on Curvature Characteristic [J]. Advances in Laser and Optoelectronics, 2017, 54(01):113-120.

9. Ruse R B, Blood N, Beetz M. Fast point feature histograms (FPFH) for 3D registration[C]. IEEE, 2009:32123217.

10. Ruse R B, Blood N, Matron Z C, et al. Aligning point cloud views using persistent feature histograms[C]. IEEE, 2008:3384-3391. 